\title{
RESEARCH ON THE VARIATION CHARACTERISTICS OF VEGETATION CHANGE AND ITS TERRAIN INFLUENCE FACTORS IN ALPINE GRASSLAND IN THE SOURCE REGION OF THE YELLOW RIVER
}

\author{
Qin Qiaoting, Chen Jianjun* \\ College of Geomatics and Geoinformation, Guilin University of Technology, Guilin 541004, \\ China-qinqiaoting@126.com (Q.Q.) chenjj@glut.edu.cn (C.J.)
}

KEY WORDS: the source region of the Yellow River; fractional vegetation coverage; pixel dichotomy model; topographic factor

\begin{abstract}
:
The source region of the Yellow River is an important ecological barrier in China. Since the 1990s, under the influence of various factors, its runoff has decreased and the ecological environment has deteriorated, which has attracted great attention of governments. Vegetation is the general term of plant communities covering the surface, which is the natural link between the atmosphere, water and soil. It plays an important role in air regulation, soil conservation and maintaining the stability of the whole ecosystem. Therefore, the study on the differential characteristics and topographic influencing factors of alpine grassland change in the source region of the Yellow River can provide reference for the ecological environment protection in -this region in the future. Based on the remote sensing data of MODIS NDVI, we studied the spatial distribution pattern and temporal variation characteristics of vegetation coverage in the source region of the Yellow River from 2000 to 2009 by means of maximum synthesis method, pixel dichotomy model method and trend analysis method. Furthermore, combined with DEM topographic elevation data, we discussed the influence of topographic factors such as elevation, slope and aspect on fractional vegetation cover (FVC) change. The results showed that: (1) From 2000 to 2009, the FVC in the source region of the Yellow River fluctuated upward, showing the spatial distribution characteristics of the southeast region $>$ the central region $>$ the northwest region; (2) During the ten years from 2000 to 2009 , the almost constant of FVC accounted for the largest area, and the areas with increasing FVC mainly concentrated in the northeast and northwest of the studied region, the areas with reduced vegetation coverage were scattered in various places in the studied region, mainly in the form of minimal patches or spots; (3) Different terrain factors had different effects on FVC and the effect of altitude on FVC changes was significantly greater than that of slope and aspect. With the increase of altitude, the proportion of significant reduction in FVC increased firstly and then decreased, while the proportion of significant increase in FVC was decreasing and the proportion of almost unchanged in FVC was increasing; With the increase of slope, the proportion in the above three cases (significant reduction, significant increase and almost unchanged in FVC) was increased, decreased gradually and fluctuated respectively. Under different aspect, the proportion in the above three cases varied little, fluctuating only within $1 \%$.
\end{abstract}

\section{INTRODUCTION}

Vegetation is the general term of plant communities covering the surface, which is explained in detail as the sum of many plants with a certain density covering the surface of a certain $\operatorname{area}(\mathrm{Xu}, 2015)$. The distribution of surface vegetation will be affected by the global ecological environment change, and then affect land use and land cover. Therefore, the ecological environment problem has been paid more and more attention by governments and scientists. The change of vegetation cover on the surface of the earth will affect the balance of regional ecosystem, which largely represents the overall state of the

\footnotetext{
* Corresponding author
} 
ecological environment and has an important impact on the global energy cycle and the biochemical cycle of matter(Jiang et al, 2015). In recent years, the environmental conditions of alpine grassland vegetation have been seriously destroyed by the influence of climate and human activities. The grassland in the source region of the Yellow River has been seriously degraded, the water conservation capacity has been reduced, and many times the flow has been cut off at the source, which seriously threatens the sustainable development of economy and society in the source region of the Yellow River and its surrounding regions, and accelerates the decline process of the alpine grassland.

In recent years, many scholars have done a lot of research on the relationship between vegetation coverage and terrain factors, but the results will vary with different research areas, different data, different research methods and different time scales(Cai et al, 2014; Xiong et al, 2018; Chen et al, 2006). Based on MODIS NDVI data of the source region of the Yellow River from 2000 to 2009, this paper estimated the fractional vegetation cover (FVC) by using the pixel dichotomy model method, studied the spatial distribution pattern and temporal variation characteristics of FVC, and combined with DEM data to analyzed the influence of topographic factors (elevation, slope, aspect) on FVC change, provide reference for the ecological environment protection in this region in the future.

\section{DATA SOURCE AND PROCESSING METHODS}

\subsection{Overview of the study area}

The source region of the Yellow River is located at $32^{\circ} 09^{\prime}$ to $36^{\circ} 33^{\prime} \mathrm{N}$ and $95^{\circ} 53^{\prime}$ to $103^{\circ} 25^{\prime} \mathrm{E}$, its northwest is adjacent to the Qaidam Basin, southwest is adjacent to the Bayan Har Mountain, Minshan Mountain and the Yangtze River Basin, and its east is separated by the Xiqishan Mountains, Taohe River and Longwu River(Li, 2011). It covers 6 states and 18 counties in Qinghai, Sichuan and Gansu provinces, with a total area of about 132,000 square kilometers. This region located in the hinterland and eastern margin of the Qinghai-Tibet Plateau, the western part of the region has typical characteristics of the Qinghai-Tibet Plateau, while the eastern part has some characteristics of the Loess Plateau(Du et al, 2015).
The source region of the Yellow River is composed of alpine, canyon, basin, glacier and other landforms, whose topography is very complex. The geomorphology of this region belongs to the Qingnan Plateau, one of the three major geomorphic units in southern Qinghai. The terrain is high in the West and low in the east, the plateau surface remains intact. The altitude distribution ranges from 2564 to $6295 \mathrm{~m}$, and the air is thin in most areas. The climate of the source region of the Yellow River belongs to the continental alpine climate of Plateau(Liu et al, 2009), which is characterized by long winter and short summer, large temperature difference between day and night, alternation of cold and hot seasons, distinct dry and wet seasons, strong solar radiation and the influence of monsoon.

\subsection{Data sources and pre-processing}

2.2.1 Data source: The 2000-2009 MODIS data and digital elevation model (DEM) data used in this paper are from the geospatial data cloud (http://www.gscloud.cn/). The MODIS MOD13Q1 image data are widely used for large-area vegetation monitoring. The data format is $\mathrm{HDF}$, the temporal resolution is 16 days and the spatial resolution is 250 meters.

2.2.2 Data preprocessing: (1) Due to the large amount of data in this study, the data is processed in batch processing. First, the MRT toolbox is used to perform format conversion, projection conversion and resample, which makes the data standard $250 \mathrm{~m}$, the format was converted to Geotiff, and the original projection was converted to Albers equal area(Chen et al, 2015). In ENVI 5.3 , the administrative vector boundaries of the source region of the Yellow River are used to tailor the data required for the study area, and the maximum synthesis method was used to process the image to reduce the impact of clouds and atmosphere.(2) The ArcGIS is used to perform mosaic and reprojection, the slope, aspect are extracted by the surface analysis function of Spatial Analysis Tools, and then reclassify the slope and aspect, the DEM is classified according to elevation, obtain elevation, slope and aspect grade distribution map of the study area.

\section{RESULT AND ANALYSIS}

\subsection{Spatial distribution characteristics of FVC}

Referring to the 《Classification Standard of Soil Erosion》(SL 
190-2007), the relevant literature(Xia et al, 2006; Zhang et al, 2015; Chen et al, 2018) and the actual situation of fractional vegetation cover $(\mathrm{FVC})$ in the source region of the Yellow River, the FVC was divided into five grades: low FVC $(<30 \%)$, medium and low FVC (30\% 45\%), medium FVC (45\% 60\%), medium and high FVC $(60 \% \sim 75 \%)$ and high FVC ( $>75 \%)$. As shown in Figure 1, the FVC in the source region of the Yellow River is generally in the middle and high level, but there are great differences among different regions, showing the distribution characteristics of the southeast region $>$ the central region $>$ the northwest region. The FVC in northwest region is relatively low, with little interannual change. The FVC in Hongyuan county, Zoige county, Jiuzhi county and Maqu county in southeast region is relatively high, with the FVC above $60 \%$, which plays a decisive role in the pattern of FVC in the source region of the Yellow River.

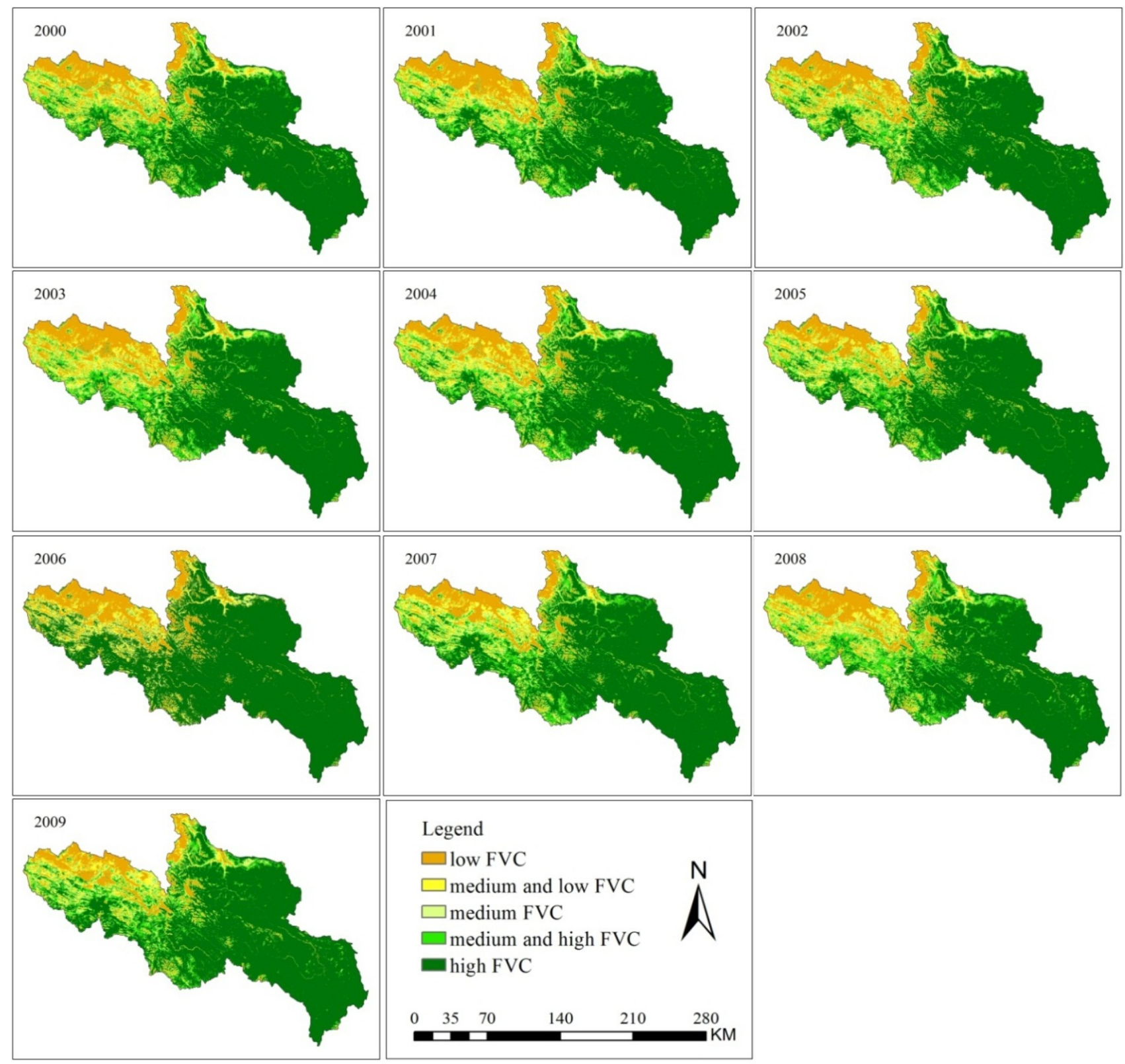

Figure 1. Monitoring results of FVC in the source region of the Yellow River from 2000 to 2009

\subsection{Temporal variation characteristics of FVC}

As shown in Figure 2, the annual average FVC in the source region of the Yellow River fluctuated between $71 \%$ and $77 \%$, and the FVC decreased gradually from 2000 to 2003, which 
indicates that the overall vegetation growth has deteriorated in recent years; the FVC fluctuated greatly from 2003 to 2009, and there is no obvious trend characteristic; the average FVC in 2008 is the lowest, only $71.10 \%$; and the FVC in 2009 increased by a large margin, reaching $76.38 \%$, up nearly $5.3 \%$ from 2008, the highest since 2000 .

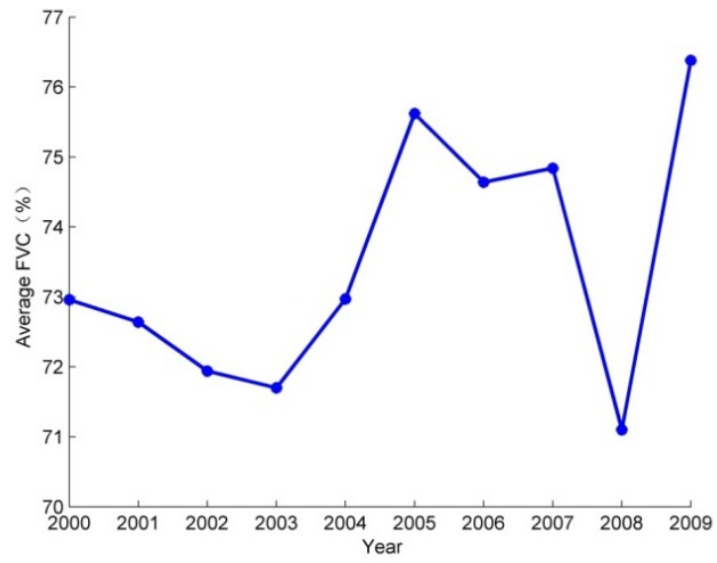

Figure 2. Changes of average FVC in the source region of the Yellow River from 2000 to 2009

\subsection{Dynamic change analysis of FVC}

\subsubsection{Trend analysis of vegetation coverage change: The} FVC in the source region of the Yellow River from 2000 to 2009 was analyzed by linear regression analysis. Based on the results of regression significance test $(\mathrm{P}<0.01)$, the change of FVC in the source region of the Yellow River was divided into three categories: significant reduction, almost unchanged and significant increase. As shown in Table 1, the areas with significant increase in FVC accounted for $16.52 \%$ of the total vegetation coverage area, the areas with almost unchanged in FVC accounted for $80.17 \%$, and the areas with significant reduction in FVC accounted for only 3.31\%.

Figure 3 shows that the increase area of FVC in the source region of the Yellow River from 2000 to 2009 is far greater than the decrease area of FVC. The areas with increasing FVC are mainly distributed in Hongyuan county, Zoige county, Maqu county, Qumali county, Mado county, Zeku county, etc. The areas with almost unchanged FVC are distributed in most areas of the source region of the Yellow River, and the areas with reduced FVC were dispersed in the source region of the Yellow River

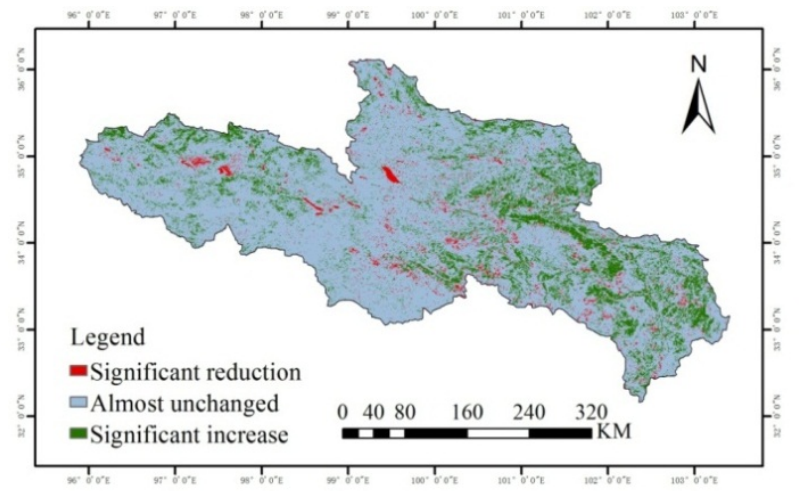

Figure 3. Trends of FVC in the source region of the Yellow River from 2000 to 2009

\begin{tabular}{ccc}
\hline Trends & Area $\left(\mathrm{km}^{2}\right)$ & Percentage $(\%)$ \\
\hline Significant reduction & 4068.562 & 3.31 \\
Almost unchanged & 98587.380 & 80.17 \\
Significant increase & 20314.690 & 16.52 \\
\hline
\end{tabular}

Table 1. Trend statistics of FVC in the source region of the

\section{Yellow River}

\subsubsection{Qualitative analysis of spatial change of vegetation} coverage: According to the slope distribution map of FVC change in the source region of the Yellow River from 2000 to 2009 (Figure 4), there are vary degree of positive and negative changes in the source region. The absolute value of the image represents the drastic degree of FVC change(Feng et al, 2014), the positive value represents the increasing trend of FVC and the negative value represents the decreasing trend. As shown in Figure 4, the areas with slope greater than 0 are mainly distributed in the northeast and northwest of the studied region, and scattered in the southeast; the areas with slope less than 0 are scattered in all parts of the studied region, mainly in the form of minimal patches or spots; on the whole, the vegetation status in the source region of the Yellow River shows an increasing trend. 


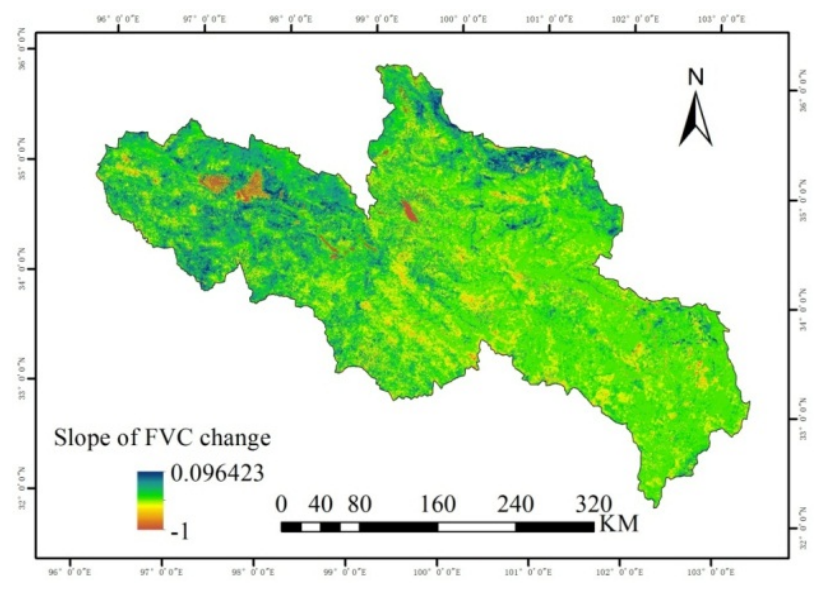

Figure 4. Slope distribution of FVC change in the source region of the Yellow River from 2000 to 2009

\subsection{Effects of terrain factors on vegetation coverage change}

\subsubsection{Effect of altitude on vegetation coverage change:}

Variations in altitude can change various environmental factors (such as temperature, humidity, light, air pressure, etc.) and thus affect the growth and development of vegetation( $\mathrm{Li}$ et al, 2016). In order to studied the relationship between FVC and altitude in the source region of the Yellow River, the altitude gradient was divided into five grades: 2564 3600m, 3600 4000m, 4000 4300m, 4300 4500m and 4500 6295m, and the change of FVC under each altitude gradient was obtained.

From Table 2, it can be seen that the proportion of high altitude areas larger than $3600 \mathrm{~m}$ in the source region of the Yellow River reaches $84.51 \%$, accounting for the vast majority of the whole basin. The change of vegetation coverage $(\mathrm{P}<0.01)$ will be different with the change of altitude. When the altitude is less than $4300 \mathrm{~m}$, the proportion of significant reduction and almost unchanged in FVC gradually increased with the increase of altitude. On the contrary, the proportion of significant increase gradually decreased with the increase of altitude. When the altitude is more than $4300 \mathrm{~m}$, the proportion of significant reduction and significant increase in $\mathrm{FVC}$ both decreased, the proportion of almost unchanged in FVC continued to rise.

\begin{tabular}{ccccc}
\hline Altitude $(\mathrm{m})$ & Area percentage $(\%)$ & $\begin{array}{c}\text { Significant } \\
\text { reduction(\%) }\end{array}$ & Almost unchanged(\%) & $\begin{array}{c}\text { Significant } \\
\text { increase }(\%)\end{array}$ \\
\hline $2564 \sim 3600$ & 15.49 & 2.72 & 66.77 & 30.51 \\
$3600 \sim 4000$ & 20.92 & 2.94 & 72.70 & 24.36 \\
$4000 \sim 4300$ & 20.80 & 5.43 & 81.25 & 13.32 \\
$4300 \sim 4500$ & 21.75 & 2.49 & 87.35 & 10.16 \\
$4500 \sim 6295$ & 21.04 & 2.84 & 89.08 & 8.08 \\
\hline
\end{tabular}

Table 2. Changes of FVC at different elevation gradients in the source region of the Yellow River

\subsubsection{Effect of slope on vegetation coverage change: Slope} represents the degree of local surface tilt, which directly affects the way and degree of surface material and energy exchange and transformation, and affects the distribution of surface vegetation by changing the basic properties of soil(OSTENDEORF et al, 1998). In this paper, the slope

\begin{tabular}{ccccc}
\hline Slope & Area percentage(\%) & $\begin{array}{c}\text { Significant } \\
\text { reduction(\%) }\end{array}$ & Almost unchanged(\%) & $\begin{array}{c}\text { Significant } \\
\text { increase(\%) }\end{array}$ \\
\hline Flat & 0.98 & 23.37 & 75.01 & 1.62 \\
Micro-slope & 13.51 & 2.48 & 79.90 & 17.62 \\
Gentle slope & 24.12 & 2.58 & 80.06 & 17.36 \\
Fastigiate slope & 34.11 & 3.07 & 79.94 & 16.99 \\
Steep slope & 18.0 & 3.70 & 80.20 & 16.10 \\
Dangerous slope & 9.28 & 4.36 & 82.21 & 13.43 \\
\hline
\end{tabular}

Table 3. Changes of FVC at different slope gradients in the source region of the Yellow River gradient of the source region of the Yellow River is divided into six grades: flat $\left(<0.001^{\circ}\right)$, micro-slope $\left(0.001^{\circ} \sim 5^{\circ}\right)$, gentle slope $\left(5^{\circ} \sim 10^{\circ}\right)$, fastigiate slope $\left(10^{\circ} \sim 20^{\circ}\right)$, steep slope $\left(20^{\circ} \sim 30^{\circ}\right)$ and dangerous slope $\left(>30^{\circ}\right)$, and the change of FVC under each slope gradient is obtained. 
From Table 3, it can be seen that the slope of the studied region is the largest in the range of $5^{\circ} \sim 20^{\circ}$, which accounting for $58.23 \%$ of the total area of the studied region. The central part of the studied region is mainly steep slope and dangerous slope. As shown in Table 3, FVC shows different trends with the increase of slope $(\mathrm{P}<0.01)$, the proportion in the three cases (significant reduction, significant increase and almost unchanged in FVC)are increased, decreased gradually and fluctuated around $80 \%$ respectively.

3.4.3 Effect of aspect on vegetation coverage change: Aspect indicates the change direction of each grid elevation change, the solar radiation and soil moisture received from different aspect are different, thus affects the distribution characteristics of vegetation(Wang et al, 2018). In this paper, the aspect is divided into nine zones: flat $\left(-1^{\circ} \sim 0^{\circ}\right)$, north $\left(0^{\circ} \sim 22.5^{\circ}\right.$ and $\left.337.5^{\circ} \sim 360^{\circ}\right)$, northeast $\left(22.5^{\circ} \sim 67.5^{\circ}\right)$, east $\left(67.5^{\circ} \sim 112.5^{\circ}\right)$, southeast $\left(112.5^{\circ} \sim 157.5^{\circ}\right)$, $\operatorname{south}\left(157.5^{\circ} \sim 202.5^{\circ}\right)$, southwest(202. $\left.5^{\circ} \sim 247.5^{\circ}\right)$,west $\left(247.5^{\circ} \sim 292.5^{\circ}\right)$, northwest $\left(292.5^{\circ} \sim 337.5^{\circ}\right)$,

\begin{tabular}{ccccc}
\hline Aspect & Area percentage(\%) & $\begin{array}{c}\text { Significant } \\
\text { reduction(\%) }\end{array}$ & $\begin{array}{c}\text { Significant } \\
\text { Almost unchanged(\%) }(\%)\end{array}$ \\
\hline Flat & 1.54 & 15.83 & 77.24 & 6.93 \\
North & 13.54 & 3.42 & 81.02 & 15.56 \\
Northeast & 13.30 & 3.34 & 80.54 & 16.12 \\
East & 10.96 & 3.14 & 80.28 & 16.58 \\
Southeast & 11.95 & 2.96 & 80.34 & 16.70 \\
South & 13.20 & 2.90 & 79.73 & 17.37 \\
Southwest & 12.57 & 2.93 & 79.31 & 17.76 \\
West & 10.73 & 2.93 & 79.96 & 17.11 \\
Northwest & 12.21 & 3.21 & 80.51 & 16.28 \\
\hline
\end{tabular}

Table 4. Changes of vegetation coverage on different aspects in the source region of the Yellow River

\section{CONCLUSION}

Through the above research results, the conclusions are as follows: (1) The FVC in the source region of the Yellow River is generally in the middle and high level, but there are great differences among different regions, showing the distribution characteristics of the southeast region $>$ the central region $>$ the northwest region. The annual average $\mathrm{FVC}$ in the source region of the Yellow River fluctuated between $71 \%$ and $77 \%$, and the FVC decreased gradually from 2000 to 2003, which indicates that the overall vegetation growth has deteriorated in recent years; the FVC fluctuated greatly from 2003 to 2009 , and there and the change of FVC under each aspect zone was obtained

As shown in Table 4, under different aspect, the proportion in the above three cases varied little, fluctuating only within $1 \%$ (only the other 8 levels except the flat were analyzed). The percentage of significant increase in FVC in the southwest was the highest, followed by the south and the west, and the smallest was the north; the highest percentage of significant reduction in FVC was the north, followed by the northeast and the northwest, and the smallest was the south; the highest percentage of almost unchanged in FVC was the north, and the smallest was the southwest. Overall, the proportion of vegetation coverage increase on sunny slope is larger than that on shady slope, and the proportion of vegetation coverage decrease is smaller than that on shady slope, because the solar radiation energy received on sunny slope and shady slope is different. 
of significant reduction in FVC increased firstly and then decreased, while the proportion of significant increase in FVC was decreasing and the proportion of almost unchanged in FVC was increasing; With the increase of slope, the proportion in the above three cases (significant reduction, significant increase and almost unchanged in FVC) was increased, decreased gradually and fluctuated respectively. Under different aspect, the proportion in the above three cases varied little, fluctuating only within $1 \%$.

\section{ACKNOWLEDGEMENTS}

This study was supported by the National Natural Science Foundation of China (41801030, 41901370, 41961065); Guangxi Natural Science Foundation (2018GXNSFBA281054, 2018GXNSFBA281075，2017GXNSFDA198016); Research Foundation of Guilin University of Technology (GUTQDJJ2017069); the BaGuiScholars program of the provincial government of Guangxi (Guoqing Zhou).

\section{REFERENCES}

$\mathrm{Xu} \mathrm{Y}, 2015$. Dynamic changes of vegetation cover and its spatial and temporal response to climate factors.

Jiang W, Yuan L, Wang W, Cao R, Zhang Y, Shen W, 2015. Spatio-temporal analysis of vegetation variation in the Yellow River Basin. Ecological Indicators. 51:117-126.

Cai H, He Z W, An Y L, Deng H, 2014. Study on the correlation between vegetation coverage and topographic factors in Chishui River Basin based on RS and GIS. Earth and Environment. 42 (04): 000518-524.

Xiong J N, Zhang H, Peng C, Fan C K, 2018. The relationship between vegetation cover change and topographic factors in Wenchuan earthquake-stricken area. Soil and Water Conservation Bulletin.

Chen Y, Xu X, Zhang D R, Wei Y, 2006. The correlation between vegetation distribution and topographic factors in northern Longmen Shanxi, Sichuan. Journal of Ecology. 25(9): 1052-1055

Li H R, 2011. Study on Water Conservation, Conservation and Development in the Source Area of the Yellow River. Yellow River Water Resources Publishing House.

Du Q, Shu J M, Xiong S S, 2015. Assessment of Climate, Vegetation Change and Water Conservation Function in the
Source Area of the Yellow River. Science Press.

Liu Q G, Chen X P, 2009. Study on the mechanism and Countermeasures of land cover change in the source area of Huanghe River based on RS/GIS. Eco-economy. (12): 54-59. Chen J, Du P J, Tan Q, 2015. Estimation and change analysis of vegetation coverage in northern China by remote sensing. Land and Resources Remote Sensing.27(2): 112-117.

SL 190-2007, Soil Erosion Classification and Classification Standard.

Xia B C, Gong J Z, 2006. Grade structure of vegetation coverage and its spatial and temporal pattern in Guangzhou. Ecology Science. 25 (5): 417-422.

Zhang H, Qu J J, Zhang K C, 2015. Extraction of remote sensing information of oasis vegetation coverage --- Taking Dunhuang oasis as an example. Desert of China. 35 (2): 493-498.

Chen L, Chen S J, Cai X B, 2018. Changes of vegetation coverage and its response to topographic factors in Hubei Province. Journal of Central South Forestry University.

Feng L L, He Z M, Liu X F, 2014. Vegetation coverage and its spatial and temporal changes in Jilin Province based on MODIS-NDVI data. Journal of the University of Chinese Academy of Sciences. 31 (4): 492-499.

Li L, Wang L, Wang X, 2016. Analysis of the effect of elevation gradient on plant growth and development. Shelterbelt Science and Technology. (5): 67-68.

OSTENDEORF B, REYNOLDS J F, 1998. Mechanism of rocky desertification derived from topographic gradients. Landscape Ecology. 7 (13): 187-201.

Wang Y, Guo Y, 2018. Spatial relationship between vegetation cover and topographic factors in Karst geomorphic areas: A case study of Puding County, Guizhou Province. Resources and Environment of the Yangtze River Basin. 